\title{
Differentiating between ADHD and ASD in childhood: some directions for practitioners
}

\author{
Nanda Rommelse ${ }^{1,2} \cdot$ Janne Visser ${ }^{2} \cdot$ Catharina Hartman $^{3}$
}

Published online: 12 May 2018

c) Springer-Verlag GmbH Germany, part of Springer Nature 2018

\section{Introduction}

Autism Spectrum Disorders (ASD) belong to a wider group of neurobiological developmental disorders, along with Attention-Deficit/Hyperactivity Disorders (ADHD) and other disorders in specific areas of development [1]. All of these disorders manifest through many common characteristics and often appear simultaneously, which can make it hard to differentiate between two or more disorders. This especially holds true for ASD and ADHD: many publications in European Child and Adolescent Psychiatry have documented on the frequent co-morbidity and resemblance in associated factors between both disorders (e.g., $[2-4,7,8]$ ). There is no diagnostic test, standardised observation or questionnaire that is specially designed to distinguish between ADHD and ASD. The question is to what extent this is a feasible or even sensible goal and has additive value for the parent or the child. A significant percentage of children does in fact show characteristics of both disorders, in which sometimes criteria for both disorders are met, but sometimes just not met $[9,10]$. The basic idea that is often employed is to examine which symptoms are the most conspicuous (regardless of which of the two disorders they belong to) and cause the most suffering and/or contribute to functional impairments. When the symptoms can largely be attributed to ASD, the legitimate question has to be asked whether it adds any value for the child and the parents to also diagnose ADHD. In our view, the answer would be: only when the ADHD symptoms

Nanda Rommelse

Nanda.Lambregts-Rommelse@ radboudumc.nl

1 Department of Psychiatry, Radboudumc, Nijmegen, The Netherlands

2 Karakter Child and Adolescent Psychiatry University Center, Nijmegen, The Netherlands

3 University of Groningen, University Medical Center Groningen, Interdisciplinary Center Psychopathology and Emotion regulation (ICPE), Groningen, The Netherlands cause significant suffering and/or lead to impairments in everyday life and, therefore, require ADHD-specific interventions. This holds equally true the other way around.

Although not set in stone, for children with a combined diagnosis ADHD + ASD, clinical practitioners usually attempt to investigate whether the hampered development of the child can better be explained from an 'ADHD perspective', an 'ASD perspective', or whether there is a clear indication of both. This is often done using (1) a semi-structured developmental interview looking for 'red flags' in early development that give a strong indication for ASD and/or ADHD and (2) a semi-structured DSM-based interview of current symptoms that appear particularly relevant in the differentiation between both disorders [13]. Below, we will illustrate both.

\section{A core component of the differential diagnostics is a semi-structured developmental interview looking for 'red flags' in early development that give a strong indication of ASD and/ or ADHD, that explicitly includes characteristics beyond the core symptoms of ADHD and ASD.}

Overlap in risk factors and early signals ( $0-4$ years of age) that are seen in both ADHD and ASD and thus do not give a direction for differential diagnostics $[5,6,11,12])$ :

- Complicated pregnancy and birth, low birth weight, prematurity, and low Apgar scores

- Self-regulation problems: excessive crying, easily upset/ overstimulated/irritated, hard to soothe/calm down, eating problems, sleep problems, overstretched posture upon holding, delayed motor development, language development, and delayed cleanliness (although some of the children with ADHD are in fact remarkably fast in these areas)

- From toddler- and preschool age: difficulties in social interaction with peers 
Early development signals ( $0-4$ years of age) that are more typical for ASD than for ADHD [6]:

- Deficits in social development: late/reduced smiling or displaying other facial expressions, little/no social gestures or 'social referencing', little/no interest in social games (peekaboo) and cuddling, little/no interest in social contact with others, little/no baby babbling, and little/no response to own name

- Atypical reactivity to sensory input: unusual strong, weak or atypical responses related to sensory stimulation (touch, smell, light, sound, pain, and movement), and repetitive motor movements (such as rocking, head banging, or twisting motions).

- Stereotyped/repetitive, restricted interests, and a preference for playing alone

- Generally lower activity level as infant (whereas often displaying increased activity from 2 to 3 years of age, like children with ADHD)

Less is known about the potential precursors of ADHD than for ASD, especially with regard to precursors that are mostly typical for ADHD and not ASD.

\section{A semi-structured DSM-based interview of current symptoms that seem particularly relevant in the differentiation between both disorders}

An important assumption is that an assessment of ASD must be based on certain combinations of symptoms (i.e., symptom profile): the presence of isolated ASD symptoms co-morbid to ADHD, such as motor stereotypies or atypical sensory responses, without social-communicative difficulties makes ASD unlikely. Similarly, isolated hyperactivity without inattention difficulties co-morbid to ASD makes an ADHD diagnosis unlikely. When a combination of ASD symptoms as listed below manifests in a child with ADHD, this strengthens the appropriateness of a co-morbid ASD and ADHD diagnosis:

- 'Deficits in nonverbal communicative behaviors used for social interaction' The use of gestures and meaningful eye contact is present in children with ADHD. However, when poorly integrated verbal and nonverbal communication, atypical eye contact and body language, deficits in understanding and use of gestures, or lack of facial expressions and nonverbal communication are present, (co-morbid) ASD should be considered.

- 'Stereotyped or repetitive motor movements, use of objects, or speech' This refers to explicit stereotypic (repetitive) movements that mainly occur when the child is excited or tries to calm itself, and not the motor restlessness typical for ADHD. Other characteristics of ASD that are atypical for ADHD are the excessive organizing of toys (instead of playing), dominance of sensory play that is not in line with developmental level such as mouthing/putting things into mouth, rhythmical moving (parts of) toys (such as turning the wheels of a car without meaning in play), (delayed) echolalia, and idiosyncratic phrases.

- 'Insistence on sameness, inflexible adherence to routines or ritualized patterns or verbal or nonverbal behavior' Changes are usually not a problem for children with ADHD, although children with ADHD without ASD can also become unsettled in new situations. The difference, however, appears to be in the nature/direction of the disruption: dysregulation due to impulsive action/reward focus that quickly may degenerate into impatience and frustration is often seen in children with ADHD, whereas fearful tension/panic for what is new/uncontrolled leading to avoidance is often seen in children with ASD. If the latter is observed in a child, (co-morbid) ASD should be considered.

- 'Highly restricted, fixated interests that are abnormal in intensity or focus' A strong attachment to-or preoccupation with-unusual objects or excessively circumscribed or perseverative interests, are not common characteristics of children with ADHD. When present, ASD may also be considered.

- 'Hyper- or hyporeactivity to sensory input or unusual interests in sensory aspects of the environment' Whenever a child appears indifferent to pain/temperature, shows adverse responses to specific sounds or textures or cannot endure these, excessively smells or touches objects and/or has a visual fascination for lights or movement, ASD should be considered, as these are not typical symptoms of ADHD.

When looking at the criteria of ADHD in a similar fashion, the descriptions given below offer some solid clues for differentiating between ASD and ADHD:

- 'Often fails to give close attention to details or makes careless errors in schoolwork, at work or during other activities' Generally, children with ASD are very focused on details and do often not miss details. In activities, an insistence on sameness and repetition is commonly seen, leading to fewer sloppy errors. Often, children with ASD are considered as too precise, in sharp contrast to children with ADHD. When carelessness and sloppy errors manifest, one should consider (co-morbid) ADHD. However, both children with ASD (excessive focus/detail-oriented) and with ADHD (inattention, very easily distracted) miss the overview. The latter is, therefore, not sufficient for a double diagnosis, nor will it be helpful in a differential diagnosis. 
- 'Often has difficulty sustaining attention in tasks or activities', and 'often unable to play or engage in leisure activities quietly' Often, children with ASD have one or more very specific and fixated interests and preoccupations in which they may engage for extended periods of time. When this does not apply and when clear difficulties in sustained attention appear, (co-morbid) ADHD should be considered. Children with ADHD can generally only focus their attention for longer periods of time when gaming or watching television. Yet, these are activities that require relatively little patience and sustained attention, as stimuli and response alternate quickly and results or rewards follow near-instantly.

- 'Often avoids, dislikes or is reluctant to engage in tasks that require sustained mental effort' Whenever tasks are predictable and not too hard, they will generally not be considered a stumbling block for children with ASD. Children with ADHD will usually get bored.

- 'Often loses things necessary for tasks or activities' Typically, most children with ASD like a fixed place for their belongings and ritualized ways of, for instance, packing their school bag.

- 'Often forgetful in daily activities' As many children with ASD without ADHD like and are focused on predictability, they will be quick to notice when things are different or forgotten.

\section{Conclusion}

The above offers some guidance in investigating whether the problems of a child with a mixed ASD + ADHD symptom profile can best be explained from an 'ADHD perspective' or an 'ASD perspective' or whether a co-morbid diagnosis offers the best fitting description. However, it is important to emphasize that an exclusive 'ADHD/ASD' perspective is too limited: in this way, many behaviors may be categorized as belonging to ASD and/or ADHD, making these diagnostic categories rapidly expanding constructs. In addition, it is likely that the core symptoms are (partly) consequences of more basic problems/mechanisms: which symptoms (mainly ASD or ADHD) are on the foreground will be a question of order of appearance or question of which competence (or limitation thereof) will be challenged first. Ideally, treatment will, therefore, be focused on the broad profile of a child: (core) symptoms, cognitive characteristics, stimuli processing, eventual emotional and behavior problems and regulation, physical health, environmental factors (protective and risk), as well as adaptive functioning. In addition to giving a much better overview of the problems and competencies of the child and subsequent interventions, a broad profile will also offer a better view on possible presence of problems other than ADHD or ASD.

\section{References}

1. American Psychiatric Association (2015) Diagnostic and statistical manual of mental disorders, 5th edn. Washington, DC

2. Andersen CH, Thomsen PH, Nohr EA et al (2018) Maternal body mass index before pregnancy as a risk factor for ADHD and autism in children. Eur Child Adolesc Psychiatry 27:139

3. Boxhoorn S, Lopez E, Schmidt C et al (2018) Attention profiles in autism spectrum disorder and subtypes of attention-deficit/ hyperactivity disorder. Eur Child Adolesc Psychiatry. https://doi. org/10.1007/s00787-018-1138-8

4. Carter Leno V, Chandler S, White P et al (2017) Testing the specificity of executive functioning impairments in adolescents with ADHD, ODD/CD and ASD. Eur Child Adolesc Psychiatry. https ://doi.org/10.1007/s00787-017-1089-5

5. Gardener H, Spiegelman D, Buka SL (2009) Prenatal risk factors for autism: comprehensive meta-analysis. Br J Psychiatry 195(1):7-14

6. Johnson MH, Gliga T, Jones E, Charman T (2015) Annual research review: infant development, autism, and ADHD - early pathways to emerging disorders. J Child Psychol Psychiatry 56(3):228-247

7. Ly V, Bottelier M, Hoekstra PJ et al (2017) Elimination diets' efficacy and mechanisms in attention deficit hyperactivity disorder and autism spectrum disorder. Eur Child Adolesc Psychiatry 26:1067

8. Muskens JB, Velders FP, Staal WG (2017) Medical comorbidities in children and adolescents with autism spectrum disorders and attention deficit hyperactivity disorders: a systematic review. Eur Child Adolesc Psychiatry 26:1093

9. Rommelse NN, Geurts HM, Franke B, Buitelaar JK, Hartman CA (2011) A review on cognitive and brain endophenotypes that may be common in autism spectrum disorder and attention-deficit/hyperactivity disorder and facilitate the search for pleiotropic genes. Neurosci Biobehav Rev 35(6):1363-1396

10. Ronald A, Larsson H, Anckarsäter H, Lichtenstein P (2014) Symptoms of autism and ADHD: a Swedish twin study examining their overlap. J Abnorm Psychol 123(2):440-451

11. Thapar A, Cooper M, Eyre O, Langley K (2013) What have we learnt about the causes of ADHD? J Child Psychol Psychiatry 54(1):3-16

12. Visser JC, Rommelse NN, Greven CU, Buitelaar JK (2016) Autism spectrum disorder and attention-deficit/hyperactivity disorder in early childhood: a review of unique and shared characteristics and developmental antecedents. Neurosci Biobehav Rev 65:229-263

13. ZERO TO THREE (2016). DC:0-5 ${ }^{\mathrm{TM}}$ : Diagnostic classification of mental health and developmental disorders of infancy and early childhood. ZERO TO THREE, Washington, DC 